\title{
Structural transformation of graphite by passage of electric current
}

Article

Accepted Version

Harris, P. J. F. (2020) Structural transformation of graphite by passage of electric current. Fullerenes, Nanotubes and Carbon Nanostructures, 28 (1). pp. 66-70. ISSN 1536-4046 doi: https://doi.org/10.1080/1536383x.2019.1671368 Available at https://centaur.reading.ac.uk/86768/

It is advisable to refer to the publisher's version if you intend to cite from the work. See Guidance on citing.

To link to this article DOI: http://dx.doi.org/10.1080/1536383x.2019.1671368

Publisher: Taylor \& Francis

All outputs in CentAUR are protected by Intellectual Property Rights law, including copyright law. Copyright and IPR is retained by the creators or other copyright holders. Terms and conditions for use of this material are defined in the End User Agreement.

\section{www.reading.ac.uk/centaur}

\section{CentAUR}

Central Archive at the University of Reading

Reading's research outputs online 


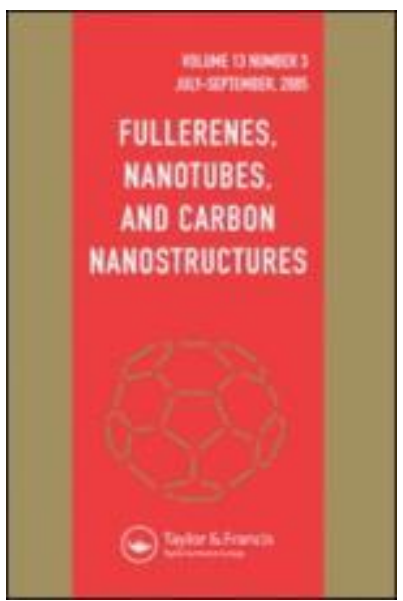

\section{Structural transformation of graphite by passage of electric current}

\begin{tabular}{|c|c|}
\hline Journal: & Fullerenes, Nanotubes and Carbon Nanostructures \\
\hline Manuscript ID & Draft \\
\hline Manuscript Type: & $\begin{array}{l}\text { Special Issue: } 13 \text { th International Conference "Advanced Carbon } \\
\text { Nanostructures" }\end{array}$ \\
\hline $\begin{array}{r}\text { Date Submitted by the } \\
\text { Author: }\end{array}$ & $\mathrm{n} / \mathrm{a}$ \\
\hline Complete List of Authors: & Harris, Peter; University of Reading - Whiteknights Campus, \\
\hline Keywords: & \\
\hline
\end{tabular}

\section{SCHOLARONE \\ Manuscripts}




\title{
Structural transformation of graphite by passage of electric current
}

\author{
Peter J.F. Harris
}

Electron Microscopy Laboratory, Department of Chemistry, J.J. Thomson Building, University of Reading, Whiteknights, Reading RG6 6AF, UK.

e-mail: p.j.f.harris@reading.ac.uk 


\section{Structural transformation of graphite by passage of electric current}

The passage of an electric current through graphite, or few-layer graphene, at high temperatures can produce dramatic structural transformations. These involve the formation of carbon structures with highly irregular morphologies, displaying many unusual features, including nanotube-graphene junctions. A number of studies of the transformed carbon have been carried out, but many aspects of its structure and formation mechanism are still not understood. This paper summarises the studies that have been made on this phenomenon, drawing mainly on the author's own work. It is shown, using high-tilt transmission electron microscopy imaging, that the transformed carbon is largely flat, and not three-dimensional as suggested in previous papers. The possible mechanism of the transformations is discussed.

Keywords: graphite; graphene; transmission electron microscopy.

\section{Introduction}

Three papers appeared within a short time of each other in 2009 which described major structural transformations of graphite or few-layer graphene produced by the passage of an electric current [1 - 3]. Two of these studies involved the in situ heating of few-layer graphite "nanoribbons" inside a transmission electron microscope (TEM) [1,2]. In both cases it was found that the graphite ribbons evolved into complex structures which largely seemed to be made up of bilayer graphene. Among the unusual features observed in the transformed carbon were single-walled nanotubes joined to bilayer graphene edges. The third study, by the present author, involved a TEM examination of bulk graphite through which a current had been passed [3]. Once again, structures with a random and complex morphology were produced, but in this case much of the material appeared to be made up of 4-layer graphene. Bilayer nanotubes joined to the graphene edges were observed.

Following this early work, several further studies of the phenomenon have been 
published [4 - 11], but many questions still remain. Thus, it is not clear whether the transformations are a result of current passing through the graphite or due to electrostatic charging of the material at high temperatures. The mechanism of the reconstruction is also unknown, with some workers interpreting the phenomenon in terms of the sublimation and edge reconstruction of the graphitic structures $[1,2,8-11]$ and others favouring a purely solid-state reconstruction mechanism [4 - 7]. A related question is whether the structures produced by these transformations are flat or threedimensional.

The primary aim of this paper is to summarise the author's own work on the effect of an electric current on the structure of graphite. The method used to pass a current through graphite is described, and typical TEM images of the transformed carbon are shown. The main part of the paper will consist of a detailed description of the structure of the carbon, gained by the application of TEM. As well as conventional bright-field and high resolution imaging, high-tilt imaging is used to determine the overall three-dimensional shape of the material. A brief summary of studies by other workers is also given. The possible mechanism of the process is considered.

\section{Experimental}

The graphite used in the experiments was a commercial synthetic graphite obtained from Quorum Technologies Ltd, UK. In order to pass a current through small samples of graphite, a commercial arc-evaporator, which is normally used for carbon-coating specimens for electron microscopy, was employed. In this unit the electrodes are graphite rods, one of which is thinned to a diameter of approximately $1.4 \mathrm{~mm}$ at the point of contact. Following evaporation, the thinned carbon rod was found to have slightly shortened, and a small deposit was formed in the area where the two rods made 
contact. This was collected and ground under isopropyl alcohol, and droplets of the suspension were pipetted onto lacey carbon TEM grids. Material collected from the fresh graphite rods was also imaged by TEM for comparison.

Conventional imaging was carried out using a JEOL 2010 microscope, with a point resolution of $0.19 \mathrm{~nm}$, operated at an accelerating voltage of $200 \mathrm{kV}$. High-tilt imaging was carried out on a JEOL 2100Plus, also operated at 200kV. This microscope has the capability of recording images at tilt values up to $\sim 80^{\circ}$. It should be noted, however, that the full range of tilt angles is only available for parts of a specimen which are positioned centrally in a grid square. Finding regions which were both correctly oriented and capable of being tilted to high angles was not easy, and it did not prove possible to carry out the kind of full tilt sequences which would be necessary in order to produce full tomographic reconstructions.

\section{Results}

A TEM image of the fresh graphite is shown in Fig. 1a. As expected, this consists mainly of flat crystallites, ranging from a few $100 \mathrm{~nm}$ to about $5 \mu \mathrm{m}$ in size, containing up to 100 layers. The carbon collected from the graphite rods following passage of a current contained some "normal" graphite, but this was accompanied by many regions which had a very different morphological appearance. A typical transformed area is shown in Fig. 1b. As can be seen, the outline of the structure of the material is more irregular than in the fresh graphite, with many curved and unusually-shaped features. A higher resolution image of the transformed carbon is shown in Fig. 1c. Two fringes can be seen along the edges of the particles in this image. This suggests that the particles are either three-dimensional, hollow, structures bounded by bilayer graphene, or flat structures with 4 graphene layers. 
Among the interesting structural features observed in the transformed carbon were nanoparticles or nanotubes apparently encapsulated inside larger structures. An image of an encapsulated bilayer nanoparticle is shown in Fig. 2a. This indicates that the larger structure must be hollow, at least near the edge. Also commonly observed were nanotubes seamlessly connected to the larger regions, as shown in Fig. 2b. A detailed analysis of these nanotube-graphene junctions has been carried out [12]. Transmission electron micrographs showed that the junction angles were not random but fell close to multiples of 30 degrees. It was demonstrated that connections with these angles are the only ones which are consistent with the symmetry of the hexagonal lattice, and molecular models showed that a continuous lattice requires the presence of large carbon rings at the junction.

High-tilt imaging of the transformed carbon was carried out in order to determine its overall three-dimensional shape. A typical tilt series is shown in Fig. 3. This shows that the region is essentially flat. However, it is clear that there are regions along the margins of the flattened particles where the graphene layers have become separated. These regions can hardly be seen in images where the flat area is perpendicular to the beam (tilt angle $+20^{\circ}$ ), but become visible as the particle is tilted. In the image recorded at a tilt angle of $-60^{\circ}$ the flattened particle is parallel to the beam, and the separated layers near the edge can be seen (arrowed). The phenomenon of expanded edges has been observed previously [6], and higher resolution images show how the 4-layer graphene sheets are opened to produce structures bounded by bilayer graphene, as in Fig. 4. 


\section{Work by other groups}

As already mentioned, a number of other groups have studied the effect of the passage of an electric current on graphite or few-layer graphene [1, 2, 8-11]. In almost all cases these studies have involved the in situ heating of few-layer graphite specimens inside a transmission electron microscope. Examples of the resulting structures are shown in Fig. 5. As in the case of the material produced from bulk graphite, we have structures with randomly-shaped edges. Short nanotubes attached to larger regions (Fig. 5(a)), or bridging two larger structures (Fig. 5(b)) can be seen. Despite the similar morphology, however, there is an important difference between these structures and those produced from bulk graphite, namely that in the former case the edges have a single fringe, while the latter generally have two fringes. If we assume that most of the material in the transformed carbons is flat, this means that the material produced in situ is largely bilayer (with single-layer nanotubes attached), while the bulk material is largely 4-layer (with bilayer nanotubes attached). It should also be noted that the nanotubes in the carbon produced by in situ heating are smaller in diameter (typically $0.8-1.5 \mathrm{~nm}$ ) than those in the bulk material $(3-4 \mathrm{~nm})$. The possible reasons for the differences between the carbons produced in the different ways are discussed below.

\section{Discussion}

The passage of an electric current, or the application of an electrostatic charge, has been widely used to modify the structure of carbon materials [13]. This paper has concentrated specifically on the remarkable structural transformations which can be produced by passing an electric current through graphite, or few-layer graphene. It has been shown, using high-tilt TEM imaging, that the transformed carbon is largely flat, and not three-dimensional as suggested in previous papers [e.g. 6]. However, threedimensional structures are present, including nanotubes seamlessly connected to the flat 
regions. Discrete nanotubes and nanoparticles are observed supported on the larger structures. It has also been shown that separated, or opened, regions occur along the margins of the flat particles.

Different theories have been put forward to explain the structural transformations described here. A number of authors have suggested that the changes involved sublimation and edge reconstruction of graphene [e.g. 1, 2]. An alternative explanation is that the transformation primarily involves a restructuring of the graphite rather than sublimation. It seems clear that the restructuring is promoted by the electric current passing through the material, or by the build-up of electrostatic charge, since simple heating of graphite to high temperatures does not produce such transformations [14]. How does the electric current, or field, induce the extreme fluidity of the carbon, which can be seen graphically in the videos which accompany reference [2]? We do not have a clear answer to this question at present. However, it is possible that the phenomenon may be related to the observation of superplasticity in nanotubes reported by Huang and colleagues in 2006 [15]. In this work, it was shown that applying a stress to single-walled carbon nanotubes (SWCNTs) through which a current is being passed can result in a remarkable elongation of up to $280 \%$. This experimental set-up is not the same as those described in this paper, in that the SWCNTs were under stress, but nevertheless it seems that the electric current helped to promote the plasticity of the carbon in a way which may be relevant to the transformations discussed here.

A striking aspect of the transformed carbon is the presence of expanded structures around the edges of the flat regions, as shown in Fig. 4. These are typically 10 $-20 \mathrm{~nm}$ in width. Sometimes nanotubes or nanoparticles are found encapsulated in these expanded regions (see Fig. 2a). It is well established that that the planes in fresh graphite often have 'closed' edges, so that the layers resemble folded sheets [e.g. 16, 
17]. The effect of the passage of an electric current seems to be to expand these closed edges. Recently, the present author and colleagues have attempted to model this phenomenon [18]. Density functional theory calculations were performed on a single folded graphene nanoribbon, applying an electric field perpendicular to the layers. We found that this resulted in electrons moving from one layer to the other, in the opposite direction to the electric field. This might result in negative charge accumulating in the bottom layer and positive charge in the top layer, These atomic charges in turn create a local field in the opposite direction of the external one, and the net forces on the atoms tend to pull the oppositely charged layers apart.

Finally I consider the possible reasons for the differences between the carbon produced by in situ heating of few-layer graphite specimens inside a transmission electron microscope and that produced from bulk graphite. It has been noted that material produced in situ is largely bilayer, while the bulk material is largely 4-layer. The key to these differences may lie in the nature of the starting materials. In the in situ work the starting materials were either few-layer graphene nanoribbons, generally produced by chemical vapor deposition, or thinned HOPG (highly orientated pyrolytic graphite). For the bulk studies the starting material was a commercial synthetic graphite. It may be that the nature of folding in these forms of graphite are different, and that this explains the differences in the transformed structures. For example, the folding in the few-layer graphene may involve single layers rather than multiple layers, as in bulk graphite. More work is needed to establish if this is the case.

\section{Acknowledgments}

I thank my colleagues at Reading, Ricardo Grau-Crespo and Victor Posligua, and Nigel Marks and Irene Suarez-Martinez of Curtin University, Australia. 


\section{References}

[1] Jia, X. T.; Hofmann, M.; Meunier, V.; Sumpter, B. G.; Campos-Delgado, J.; Romo-Herrera, J. M.; Son, H.; Hsieh, Y. P.; Reina, A.; Kong, J.; Terrones, M.; Dresselhaus, M. S. Controlled formation of sharp zigzag and armchair edges in graphitic nanoribbons. Science 2009, 323 1701-1705. DOI:

10.1126/science. 1166862 .

[2] Huang, J. Y.; Ding F.; Yakobson, B. I.; Lu, P.; Qi, L.; Li, J. In situ observation of graphene sublimation and multi-layer edge reconstructions. Proceedings of the National Academy of Sciences of the United States of America 2009, 106, 10103-10108. DOI: 10.1073/pnas.0905193106.

[3] Harris, P. J. F. Ultrathin graphitic structures and carbon nanotubes in a purified synthetic graphite. Journal of Physics: Condensed Matter 2009, 21, 355009. DOI: $10.1088 / 0953-8984 / 21 / 35 / 355009$.

[4] Harris, P. J. F. Structural transformation of graphite by arc-discharge.

Philosophical Magazine 2011, 91, 2355-2363. DOI:

10.1080/14786435.2011.562250.

[5] Harris, P. J. F. Hollow structures with bilayer graphene walls. Carbon 2012, 50, 3195-3199. DOI: 10.1016/j.carbon.2011.10.050.

[6] Harris, P. J. F.; Slater, T. J. A.; Haigh, S. J.; Hage, F. S.; Kepaptsoglou, D. M.; Ramasse, Q. M.; R. Brydson. Bilayer graphene formed by passage of current through graphite: evidence for a three-dimensional structure. Nanotechnology 2014, 25, 465601. DOI: 10.1088/0957-4484/25/46/465601.

[7] Harris, P. J. F. Structural transformation of natural graphite by passage of an electric current. Carbon 2016, 107, 132-137. DOI:

10.1016/j.carbon.2016.05.064.

[8] Qi, L.; Huang, J.Y.; Feng, J.; Li, J. In situ observations of the nucleation and growth of atomically sharp graphene bilayer edges. Carbon 2010, 48, 23542360. DOI: 10.1016/j.carbon.2010.03.018. 
[9] Qi, J. S.; Huang, J.Y.; Feng, J.; Shi, D. N.; J. Li. The possibility of chemically inert, graphene-based all-carbon electronic devices with $0.8 \mathrm{eV}$ gap. ACS Nano 2011, 5, 3475-3482. DOI: 10.1021/nn102322s.

[10] Barreiro, A.; Börrnert, F.; Rümmeli, M. H.; Büchner, B.; Vandersypen, L. M. K. Graphene at high bias: Cracking, layer by layer sublimation, and fusing. Nano Letters 2012, 12, 1873-1878. DOI: 10.1021/n1204236u.

[11] Cruz-Silva, E.; Jia, X. T.; Terrones, H.; Sumpter, B. G.; Terrones, M.; Dresselhaus, M. S.; Meunier, V. Edge-edge interactions in stacked graphene nanoplatelets. ACS Nano 2013, 7, 2834-2841. DOI: 10.1021/nn4004204

[12] Harris, P. J. F.; Suarez, I.; Marks, N.A. The structure of junctions between carbon nanotubes and graphene shells. Nanoscale 2016, 8 , 18849. DOI: 10.1039/c6nr06461b.

[13] Harris, P. J. F. Engineering carbon materials with electricity. Carbon 2017, 122, 504-514. DOI: 10.1016/j.carbon.2017.06.084.

[14] Campos-Delgado, J.; Kim, Y. A.; Hayashi, T.; Morelos-Gómez, A.; Hofmann, M.; Muramatsu, H.; Endo, M.; Terrones, H.; Shull, R. D.; Dresselhaus, M. S.; Terrones, M. Thermal stability studies of CVD-grown graphene nanoribbons: Defect annealing and loop formation. Chem. Phys. Lett. 2009, 469, 177-182. DOI: 10.1016/j.cplett.2008.12.082.

[15] Huang, J. Y.; Chen, S.; Wang, Z. Q.; Kempa, K.; Wang, Y. M.; Jo, S.H.; Chen, G.; Dresselhaus, M. S.; Ren, Z. F. Superplastic carbon nanotubes. Nature 2006, 439, 281. DOI: 10.1038/439281a.

[16] Rotkin, S.V.; Gogotsi, Y. Analysis of non-planar graphitic structures: from arched edge planes of graphite crystals to nanotubes. Materials Research Innovations 2002, 5, 191-200. DOI: 10.1007/s10019-001-0152-4.

[17] Liu, Z.; Suenaga, K.; Harris, P. J. F.; Iijima, S. Open and closed edges of graphene layers. Physics Rev. Letters 2009, 102, 015501. DOI: 10.1103/PhysRevLett.102.015501. 
[18] Posligua, V.; Bustamante, J.; Zambrano, C. H.; Harris, P. J. F.; Grau-Crespo, R. The closed-edge structure of graphite and the effect of electrostatic charging, in the press (2019).

\section{Figure captions}

Fig. 1 (a) Transmission electron micrograph of carbon from fresh graphite rod. (b) Region with disordered structure following passage of current. (c) High resolution image of transformed carbon showing double fringes along the edges of the particles.

Fig. 2 Unusual structures in graphite transformed by exposure to an electric field. (a) Apparently hollow structure with bilayer nanoparticle inside, (b) bilayer nanotube joined to larger graphene structure. Reproduced with permission from ref [7].

Fig. 3 TEM images of transformed graphite at a range of tilt values. Arrow at top right shows direction of tilt. Arrow in $-60^{\circ}$ image shows expanded region at edge.

Fig. 4 HRTEM image showing structure of expanded edge in transformed graphite.

Fig. 5 Structures produced by in situ passage of current through graphitic nanoribbon inside a TEM. (a) Images from the work of Huang et al. [2]. The numbers 4 and 5 in this figure indicate regions which the authors believe to contain 4 and 5 bilayers, respectively. The hexagon marks the orientation of the 6 equivalent $\{1100\}$ planes. (b) Images from the work of Qi et al. [9]. 

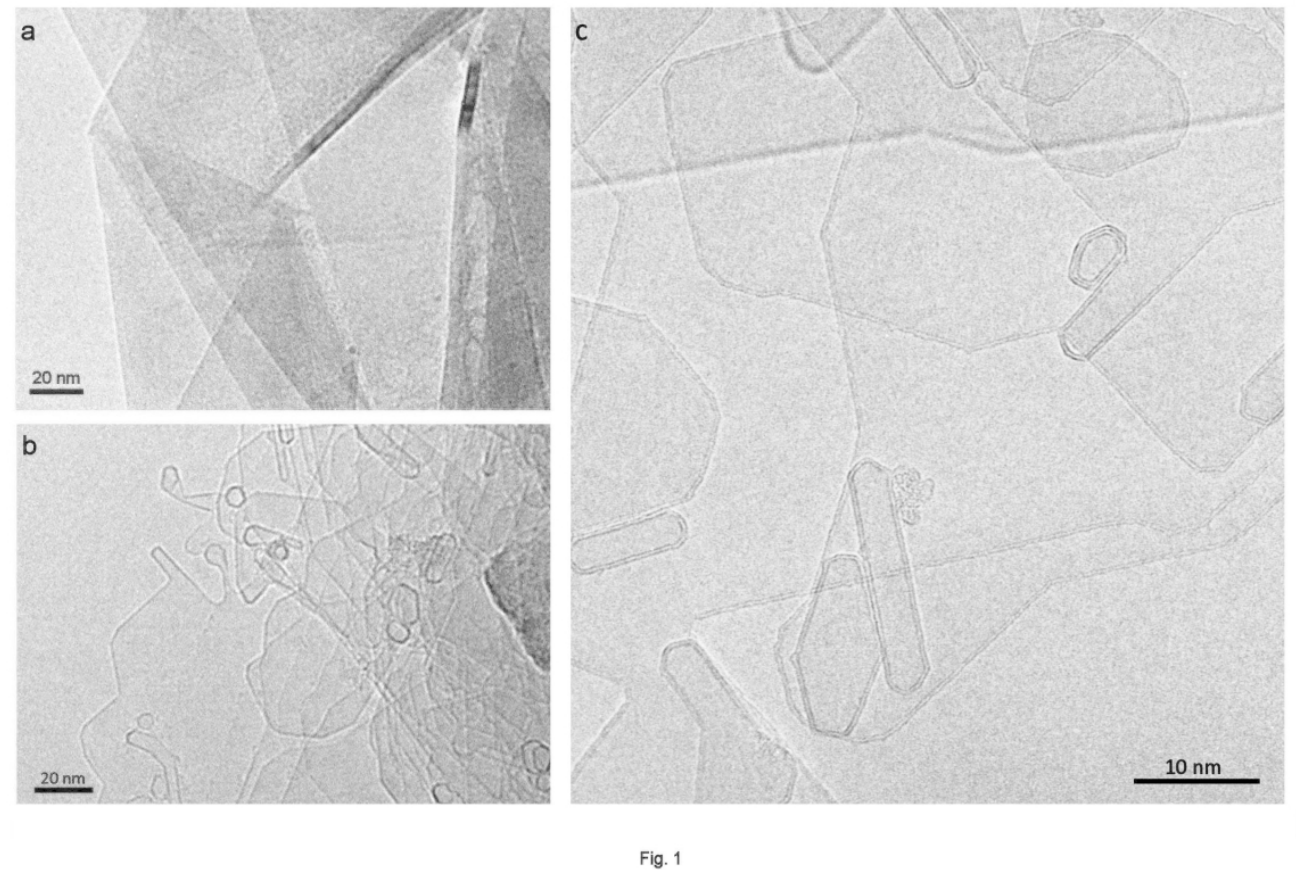

(a) Transmission electron micrograph of carbon from fresh graphite rod. (b) Region with disordered structure following passage of current. (c) High resolution image of transformed carbon showing double fringes along the edges of the particles.

$205 \times 141 \mathrm{~mm}(300 \times 300 \mathrm{DPI})$ 

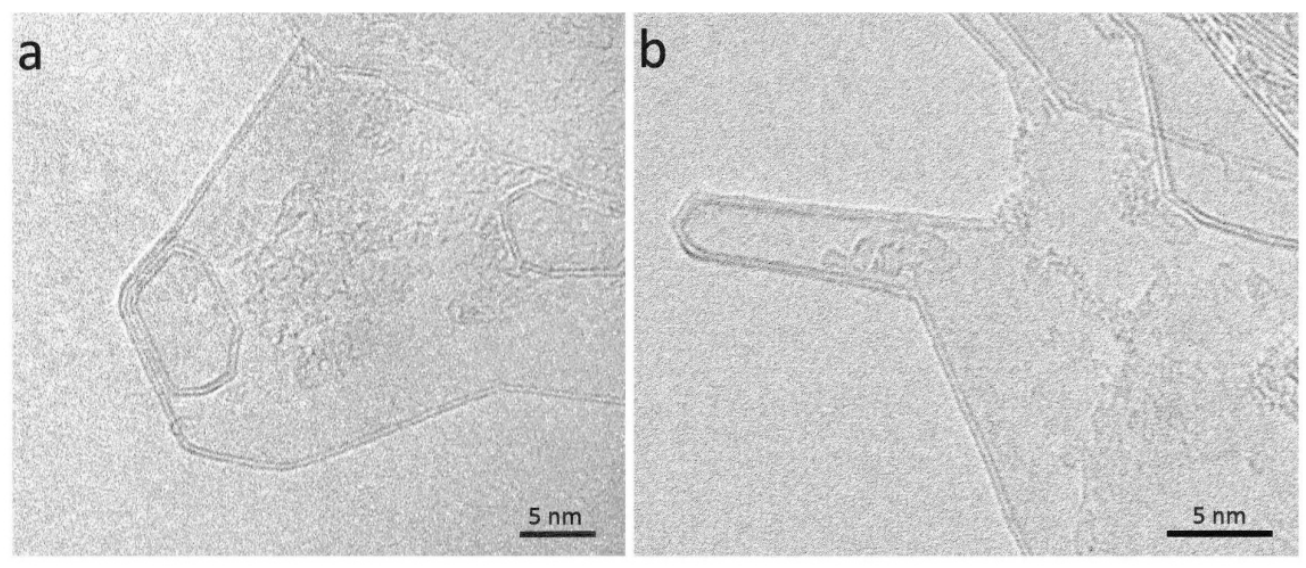

Unusual structures in graphite transformed by exposure to an electric field. (a) Apparently hollow structure with bilayer nanoparticle inside, (b) bilayer nanotube joined to larger graphene structure. Reproduced with permission from ref [7].

$160 \times 69 \mathrm{~mm}(300 \times 300 \mathrm{DPI})$ 

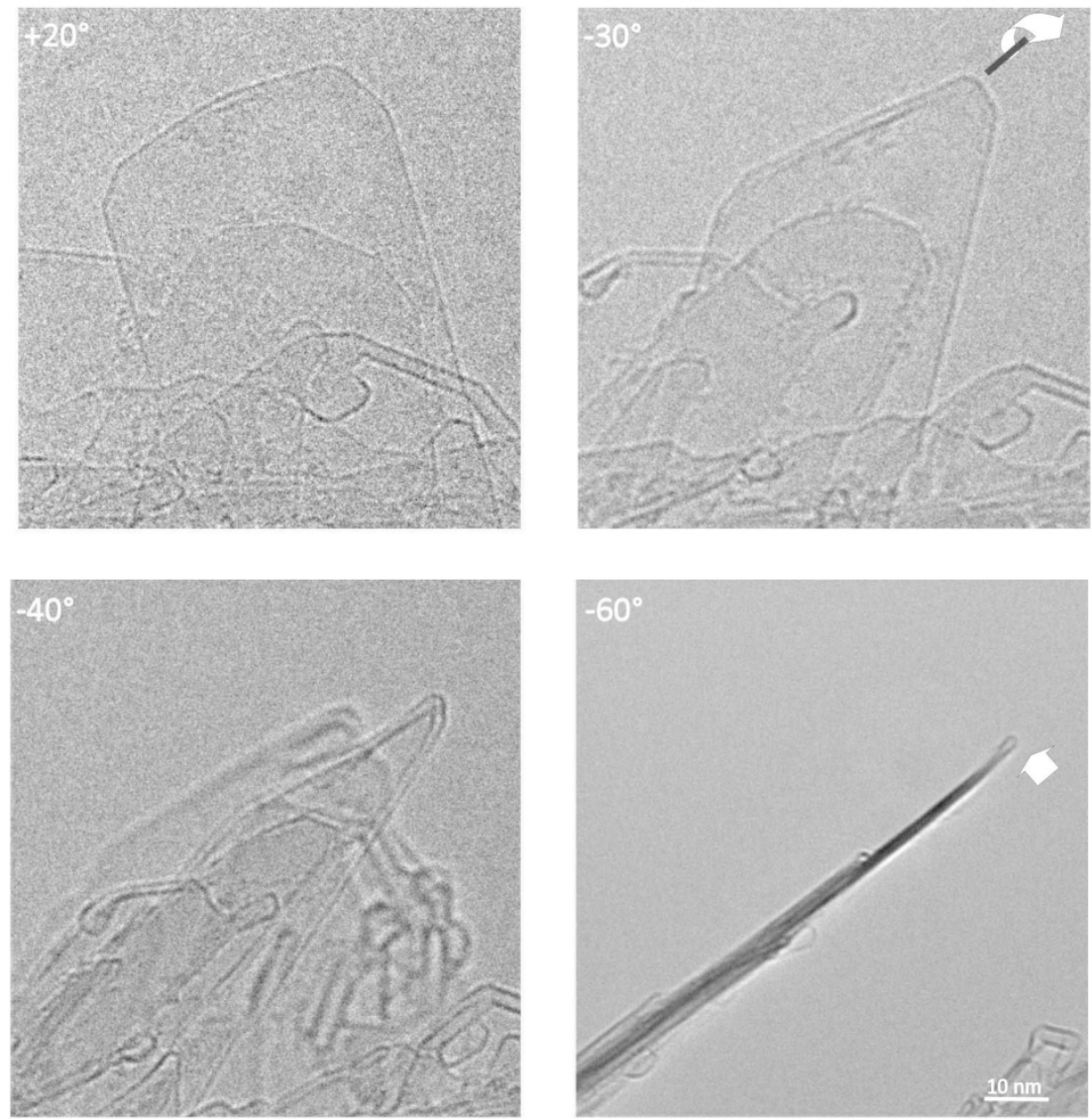

TEM images of transformed graphite at a range of tilt values. Arrow at top right shows direction of tilt. Arrow in $-60^{\circ}$ image shows expanded region at edge.

$230 \times 256 \mathrm{~mm}(198 \times 198 \mathrm{DPI})$ 


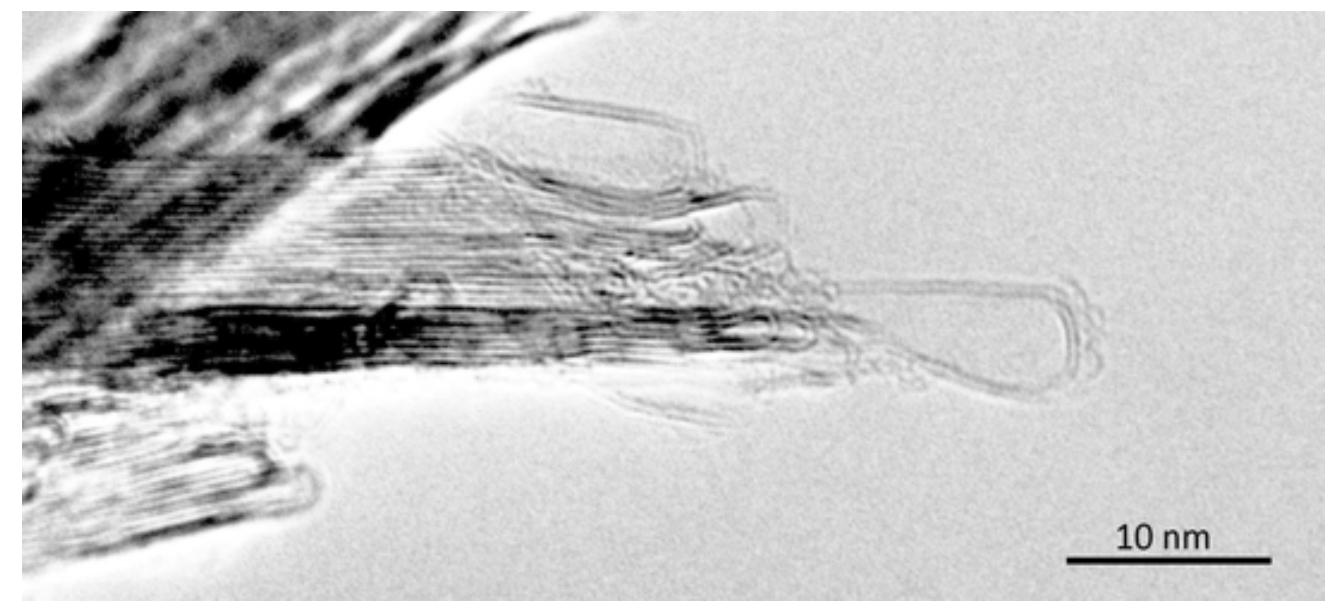

HRTEM image showing structure of expanded edge in transformed graphite.

$23 \times 10 \mathrm{~mm}(600 \times 600 \mathrm{DPI})$ 

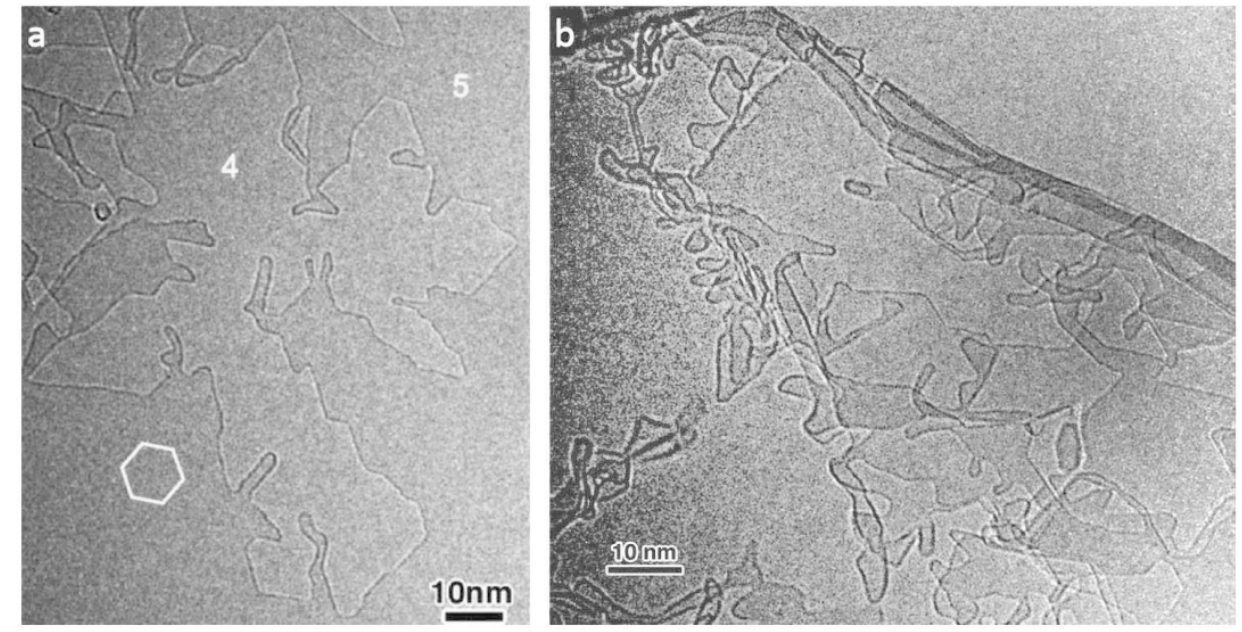

Structures produced by in situ passage of current through graphitic nanoribbon inside a TEM. (a) Images from the work of Huang et al. [2]. The numbers 4 and 5 in this figure indicate regions which the authors believe to contain 4 and 5 bilayers, respectively. The hexagon marks the orientation of the 6 equivalent $\{1100\}$ planes. (b) Image from the work of Qi et al. [9].

$$
202 \times 111 \mathrm{~mm}(198 \times 198 \text { DPI })
$$

